Topics

\title{
Performance Evaluation of Nano-JASMINE Telescope Flight Model
}

\author{
By Yoshito NiwA ${ }^{1)}$, Yoichi Hatsutori ${ }^{1)}$, Junpei MurookA ${ }^{2)}$, Yukiyasu KobAYASHI ${ }^{1)}$, Naoteru GoudA ${ }^{1)}$, Taihei YANO ${ }^{1)}$, \\ and Yoshiyuki YAMADA ${ }^{3)}$ \\ ${ }^{1)}$ National Astronomical Observatory, Tokyo, Japan \\ ${ }^{2)}$ University of Tokyo, Tokyo, Japan \\ ${ }^{3)}$ Kyoto University, Kyoto, Japan \\ (Received June 27th, 2011)
}

\begin{abstract}
The space astrometry satellite, Nano-JASMINE (Japan Astrometry Satellite Mission for Infrared Exploration), is a 5 $\mathrm{cm}$ diameter telescope designed to be sensitive to wavelength of $\mathrm{zW}$-band $(600<\lambda<1000 \mathrm{~nm})$ and to achieve astrometry measurements of nearby bright stars ( $\mathrm{zw}<7.5 \mathrm{mag}$ ) with an accuracy of 3 milli-arcseconds. This satellite will be launched by Cyclone-4 rocket from Brazil in 2013. A mission system flight model has been fabricated, and the performance has been evaluated; wave front errors were measured for the telescope mirrors and a radiation damage test was performed for a fully depleted charge coupled device image sensor used as a detector. This paper reports results of such a performance evaluation.
\end{abstract}

Key Words: Astronomy, Small Satellites, Reflecting Telescope, Techniques and Instrumentation

\section{Introduction}

Nano-JASMINE (Japan Astrometry Satellite Mission for Infrared Exploration) is a very small, 35-kilogram mass, satellite for space astrometry ${ }^{1)}$, launched by Cyclone-4 rocket from Brazil in 2013. The satellite has been developed by the National Astronomical Observatory of Japan, Kyoto University and the University of Tokyo. Nano-JASMINE is equipped with a 5-cm effective diameter telescope and a time-delay integration (TDI) controlled fully depleted charge coupled device (FDCCD) image sensor which is sensitive to wavelength of zw-band $(600<\lambda<1000 \mathrm{~nm})$. Nano-JASMINE will perform first-time demonstrations and experimentations for Japan astrometry mission in space. These trials are targeted toward the JASMINE ${ }^{2)}$. On the Nano-JASMINE mission, we are also going to measure positions of ten or twenty thousands of stars of $\mathrm{zw}<7.5 \mathrm{mag}$ for all-sky with an accuracy of about 3 milli-arcseconds.

Astrometry is a field of observational astrophysics that measures positions and proper motions of stars on the celestial sphere, and determines the distances to stars from the earth using trigonometric parallaxes due to earth orbit around the sun. Space telescopes for astrometry have significant advantages over ground-based telescopes; because of the effect of atmospheric motions, observed star images are blurry in the case of ground-based observations. Therefore, HIPPARCOS (High Precision Parallax Collecting Satellite) was launched by ESA in 1989, and observed hundreds of thousands of star positions around the all-sky with one milli-arcsecond angular accuracy ${ }^{3)}$. In 2013, almost 24 years have passed since the end of the HIPPARCOS mission. By this time, errors in proper motions have accumulated over the years; the individual motions of stars and their uncertainties are large enough to degrade the HIPPARCOS data during these two decades of blank. Therefore, the Nano-JASMINE mission is beneficial.

Currently, satellite functional tests, which include electrical integration tests, vibration tests, thermal vacuum tests, and radiation tests, have almost finished, and long duration operational tests are in the process of preparation for the launch in December 2013. In this paper, we report results of the mission system performance evaluation: a measurement of wave front error for the telescope and a radiation damage test of the FDCCD detector.

\section{Nano-JASMINE}

\subsection{Satellite overview}

Specifications for the Nano-JASMINE satellite are shown in Table 1 . The spacecraft's size is $508 \times 508 \times 504 \mathrm{~mm}$ : almost a cube, with a mass of $35 \mathrm{~kg}$ (separation mechanism is not included) (a flight model is shown in Fig. 1). The orbit is sun-synchronous orbit. This orbit has been selected because thermal stability is needed around mission system with a level of $0.1 \mathrm{~K}$; in particular, a beam-combiner, which is a part of the telescope, requires $1 \mathrm{mK}$. To achieve a mission goal the attitude of the spacecraft must be controlled with a high accuracy. Requirements are shown in Table 2. This is indeed a challenging problem for current small satellites. Therefore, Nano-JASMINE is equipped with many types of attitude sensors having different levels of accuracy, including a star tracker, fiber optical gyroscope, silicon MEMS (Micro Electro Mechanical Systems) gyroscope, sun sensor, as well as coarse and fine magnetic sensors. The measurement accuracy of each sensor is shown in Table 3. In addition, attitude errors are extracted from the shapes of the stellar images observed by the mission's telescope, which can be the most accurate sensor; when the spacecraft attitude is not stable, the shape of observed stellar image is extended relative to that of ideal one, and the amount of extension increase with the square of the 
error of angular velocity ${ }^{4}$. Magnetic torquers and reaction wheels are used as satellite attitude actuators, and 3-axis attitude control will be achieved.

Table 1. The specifications of the satellite.

\begin{tabular}{|l|l|}
\hline Size & $508 \times 508 \times 504 \mathrm{~mm}$ \\
\hline Weight & $35 \mathrm{~kg}$ \\
\hline Orbit & Sun-synchronous orbit \\
\hline Mission life time & 2 years \\
\hline Altitude & $600 \sim 1000 \mathrm{~km}$ \\
\hline Satellite communication & S-band $/ 100 \mathrm{kbps}$ \\
\hline
\end{tabular}

Table 2. The requirements for an attitude control system.

\begin{tabular}{|l|l|}
\hline $\begin{array}{l}\text { Accuracy of attitude } \\
\text { determination }\end{array}$ & $0.05 \mathrm{deg}$ \\
\hline Attitude stability & $740 \mathrm{milli}-$ arcseconds $/ 8.8 \mathrm{sec}$ \\
\hline Spin alignment & $0.05 \mathrm{deg}$ \\
\hline
\end{tabular}

Table 3. The measurement accuracy of each sensor.

\begin{tabular}{|l|l|}
\hline \multicolumn{1}{|c|}{ Sensor } & \multicolumn{1}{c|}{ Measurement accuracy } \\
\hline Coarse magnetic sensor & $1000 \mathrm{nT}$ \\
\hline Fine magnetic sensor & $200 \mathrm{nT}$ \\
\hline Sun sensor & $1 \mathrm{deg}$ \\
\hline Silicon MEMS gyroscope & $0.1 \mathrm{deg} / \mathrm{sec}$ \\
\hline Star tracker & $50 \mathrm{arcseconds}$ \\
\hline Fiber optical gyroscope & $0.2 \mathrm{arcseconds} / \mathrm{sec}$ \\
\hline
\end{tabular}

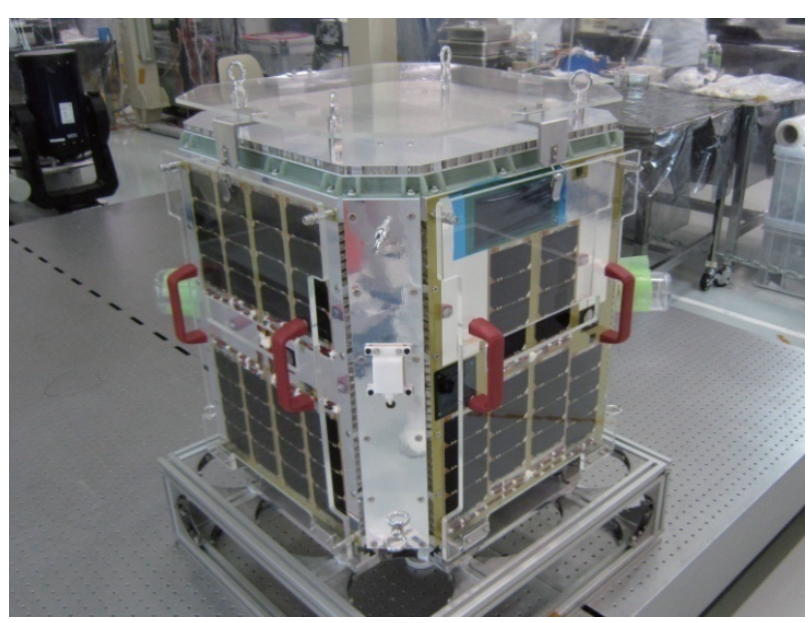

Fig. 1. The flight model of Nano-JASMINE.

\subsection{Mission sequence}

A mission sequence diagram is shown in Fig. 2. After launch, firstly, the satellite is operated in critical phase for 1 week. In this phase, an initial check is performed for the spacecraft system to confirm that the system work properly.

Secondly, the operation moves on to the next phase: early operation phase. In this phase, the mission equipments are verified, and observation is demonstrated for durations of 3 weeks. Then, the operation passes into normal phase. In this phase, maintaining high attitude and thermal stability, the satellite operates in an astrometric observation mode for more than $90 \%$ of the mission durations of 2 years.

\subsection{Mission system}

The mission system consists of two components; one is the telescope, and the other is the detector. The specifications of the mission system are shown in Table 4. The telescope is a Ritchey-Chretien type telescope with a primary mirror $5 \mathrm{~cm}$ in diameter and consists of beam-combiner, two hyperboloid mirrors and three flat mirrors ${ }^{5)}$. Figures 3 and 4 show the flight model of the mirrors and the assembled telescope, respectively.

The telescope and telescope structure are made entirely of aluminum to reduce thermal distortion. The mirrors are physically deposited with $\mathrm{Cr}$ and $\mathrm{Au}$ vapor so that the mission system has high sensitivity $(>70 \%)$ in $z w-b a n d$. The beam-combiner that combines two fields separated by 99.5 degrees is located in front of the main telescope optics. Focal length is determined from the diffraction size, which becomes equal to an area covered by two or more pixels at the focal plane in order to determine centroids of star images.

At the focal plane of the telescope, the FDCCD detector developed by Hamamatsu Photonics Co. $\mathrm{Ltd}^{6}$ ) is placed. In the red wavelength region, the response of the FDCCD detector is much better than that of a conventional CCD detector. The size of the FDCCD detector is $2 \mathrm{~K} \times 1 \mathrm{~K}$ pixels, and we use the $1 \mathrm{~K} \times 1 \mathrm{~K}$ portion of the FDCCD detector for the observations.

The total field of view of the telescope is $0.5 \times 0.5$ degrees. The pixel size is 15 micrometers, which corresponds to 1.8 arcseconds of the sky, and smaller than half the size of the radius of the Airy disk for the center wavelength of $800 \mathrm{~nm}$ so that the pixel size satisfies the Nyquist sampling condition. A combination of the FDCCD detector response and the long-pass filter, which is cut off wavelength of 0.6 micrometer, defines the zw-band filter. The FDCCD detector is controlled with the time-delay integration mode by an electronic circuit, which makes the charge transfer rate synchronize satellite spin velocity so that the FDCCD detector can detect many photons from each star in one readout cycle. Thus, readout noises can be reduced.

Fig. 2. The mission sequence diagram of Nano-JASMINE.

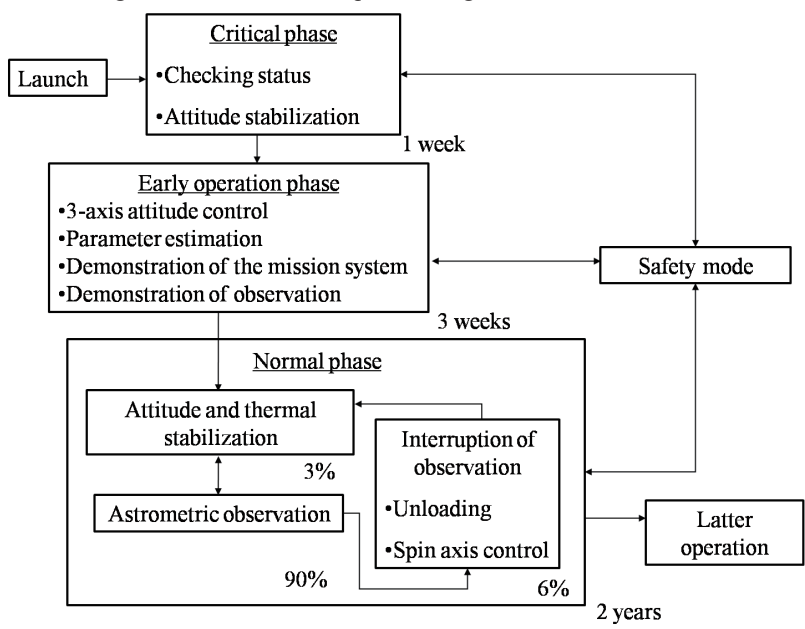


Table 4. The specifications of the mission system.

\begin{tabular}{|l|l|l|}
\hline Telescope & Type & Ritchey-Chretien \\
\cline { 2 - 3 } & Aperture & $5 \mathrm{~cm}$ \\
\cline { 2 - 3 } & Focal length & $1.67 \mathrm{~m}(\mathrm{~F} / 33)$ \\
\cline { 2 - 3 } & Field of view & $0.5 \times 0.5 \mathrm{deg}$ \\
\cline { 2 - 3 } & Basic angle & $99.5 \mathrm{deg}$ \\
\cline { 2 - 3 } & $\begin{array}{l}\text { Observation } \\
\text { wavelength }\end{array}$ & $\begin{array}{l}\text { zw-band } \\
(600 \sim 1000 \mathrm{~nm})\end{array}$ \\
\hline Detector & Type & $\begin{array}{l}\text { FDCCD (Time delayed } \\
\text { integration })\end{array}$ \\
\cline { 2 - 3 } & Pixel size & $15 \mu \mathrm{m}$ \\
\cline { 2 - 3 } & Number of pixels & $1024 \times 1152$ \\
\cline { 2 - 3 } & $\begin{array}{l}\text { Operation } \\
\text { temperature }\end{array}$ & $173 \mathrm{~K} \sim 223 \mathrm{~K}$ \\
\hline
\end{tabular}

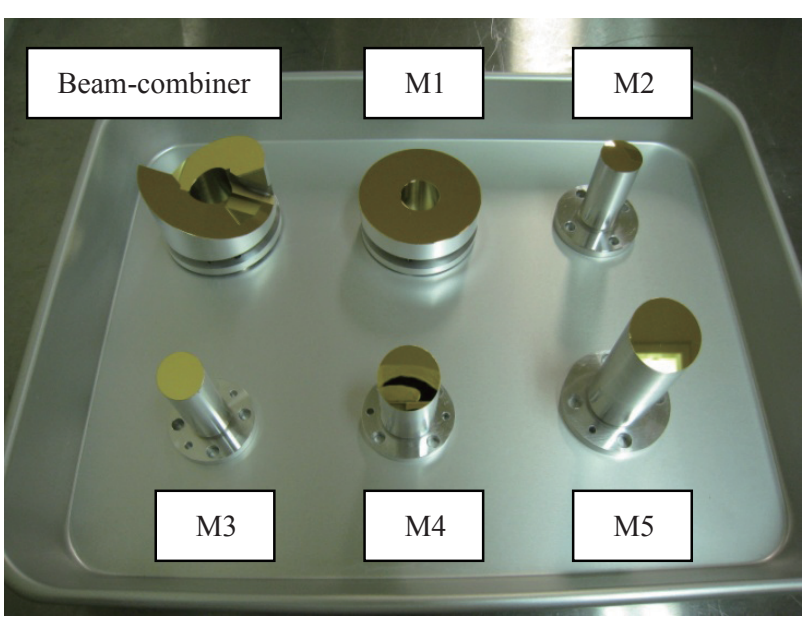

Fig. 3. The flight model of the telescope mirrors; M1 is a hyperboloid mirror as a primary mirror, M2 is a hyperboloid mirror as a secondary mirror, M3 M4 are flat mirrors.

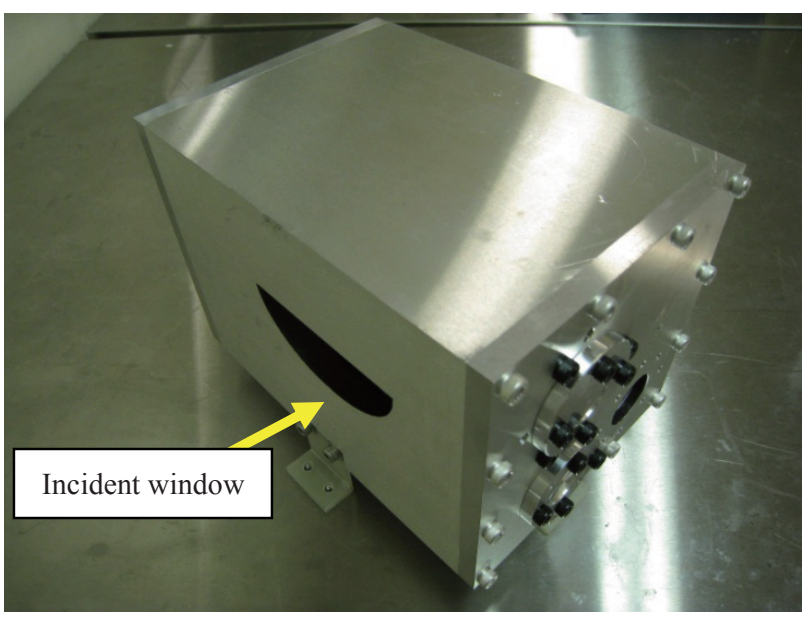

Fig. 4. The assembled flight model of the telescope. It totally weighs $1.7 \mathrm{~kg}$ and occupies a volume of $17 \times 12 \times 12 \mathrm{~cm}$.

\section{Evaluation and Results}

\subsection{Telescope}

If there are mirror surface errors for the telescope, such errors cause optical aberrations. Accordingly, shapes of point spread functions (PSF) are distorted, and the centroids of stellar images will be changed from true positions. A requirement for the total root mean square (RMS) wave front error of the telescope system is $\lambda / 14$ at $\lambda=800 \mathrm{~nm}$, which is given by the Marechal criterion ${ }^{7)}$.

The RMS wave front error was measured for each mirror surface by use of an interferometer (Zygo, GPI-XP). The results are shown in Table 5. The root sum of squares (RSS) of the RMS wave front errors of all mirrors are both $\lambda / 17$ at $\lambda$ $=800 \mathrm{~nm}$ for upper and lower optical systems. We also directly measured total wave front error for the fabricated telescope, which was placed in a vacuum chamber and cooled downed to $223 \mathrm{~K}$, which is equal to the operating temperature on the orbit. As a result, the RMS wave front errors of the telescope system are $\lambda / 19$ and $\lambda / 21$ for upper and lower optical system, respectively. This result indicates that the error level fulfills the requirement so that the telescope is given assurance for diffraction-limited performance on the orbit.

Table 5. RMS wave front error of each mirror surface.

\begin{tabular}{|c|c|c|}
\hline Mirror & RMS Wav & ror (nm) \\
\hline Beam combiner & $\begin{array}{c}\text { Upper } \\
16.6\end{array}$ & $\begin{array}{c}\text { Lower } \\
14.2\end{array}$ \\
\hline $1 \mathrm{st}$ & \multicolumn{2}{|c|}{33.2} \\
\hline 2nd & \multicolumn{2}{|c|}{20.6} \\
\hline $3 r d$ & \multicolumn{2}{|c|}{12.7} \\
\hline 4 th & \multicolumn{2}{|c|}{11.1} \\
\hline 5 th & \multicolumn{2}{|c|}{13.5} \\
\hline Total & 47.4 & 46.9 \\
\hline
\end{tabular}

\subsection{Detector}

Space radiation damage is one of the most important considerations for using semiconducting devices in an orbit ${ }^{8)}$.

The damage is classified into two types; one is ionization damage, and the other is bulk damage. Ionization damage causes an increase in dark-current, and it affects Nano-JASMINE observation as an increase in dark noise of the FDCCD detector. As for bulk damage, it causes a lattice defect so that an electron can be trapped while being transferred on the FDCCD detector, and the FDCCD detector's charge transfer efficiency (CTE) becomes degraded. Therefore, if the FDCCD detector is affected by such radiation damage, a PSF will be changed, and the positions of the stellar images will not be determined with high accuracy. To estimate an impact for the Nano-JASMINE mission, radiation experiments are needed for the FDCCD detector.

Effective radiation particle is proton; it is so abundant compared to other elements. Then, a total dose test was performed with proton beams by use of a tandem accelerator at Kyoto University. The total dose of proton beam irradiation was about $7.7 \times 10^{9}$ protons $(10 \mathrm{MeV}) \mathrm{cm}^{-2}$, which was calculated utilizing the space environment information system $\left(\right.$ SPENVIS) ${ }^{9)}$ under specified conditions: sun-synchronous orbit with altitude of $800 \mathrm{~km}$, solar maximum, and 2 years mission life.

After proton beam irradiation, the FDCCD detector was 
cool downed to $223 \mathrm{~K}$, and the dark noise level of the FDCCD detector was measured with an integral time of 8 seconds, which is equal to one of the mission operations. Figure 5 shows a comparison of a noise level of the FDCCD detector before and after proton beam irradiation. Red and green points indicate the noise level of the FDCCD detector before and after proton beam irradiation, respectively. These noise levels correspond to sum of a dark current noise and a readout noise level. The dark current noise level can be calculated by subtracting a noise level of an overscan area, which corresponds to the readout noise level. As a result, we obtained a dark current nose level before and after proton beam irradiation of 4 and 16 electrons, respectively; the noise level had quadrupled.

We also measured the CTE by using a Fe-55 X-ray source $^{10)}$, and evaluated the effect of proton radiation. As for an original CTE, a value of $>0.999995$ had been measured. A result is shown in Fig. 6. According to the increasing number of transfer, the amount of signal charge loss increases. A fitting line for a set of data points is defined as $\mathrm{f}(\mathrm{x})=\mathrm{A} \times \mathrm{B}^{\mathrm{x}}$; $\mathrm{f}(\mathrm{x})$ is called a single-pixel-event line, where $\mathrm{x}$ is number of transfer, and parameter B corresponds to a value of a CTE.

Then, a value of 0.99983 is obtained. It is confirmed that the CTE changes for the worse.

From these results, the effect on the accuracy of measuring stellar positions is estimated by error calculation; it is assumed that trapped electrons are released after other electrons are transferred more than 10 pixels away. We have learned that the value of the accuracy which we can achieve by 2 years observation is 3.22 milli-arcseconds; this value becomes worse compared to an original estimated value of 2.67 milli-arcseconds. But this effect is not critical for the mission; so that we are able to make a decision about using this FDCCD detector as the flight model.

\section{Conclusions}

We reported the results of performance evaluation of the flight model of mission system for Nano-JASMINE. In terms of the performance of the telescope, the total RMS wave front errors are $\lambda / 19$ and $\lambda / 21$ for upper and lower optical systems, respectively. This result indicates that the error level fulfills the requirement of $\lambda / 14$. In regard to the performance of the detector, the FDCCD detector can be affected by space radiation; the dark noise increases and the CTE becomes worse, but the effects are not critical for the mission. As a result, we make a decision about using this mission system as the flight model of it.

\section{Acknowledgments}

The practical machining processes for optical components in this study were carried out by colleagues at the Corporate Manufacturing Engineering Center of TOSHIBA Co. Ltd. Some mission parts of the flight model are made by the Advanced Technology Center at the National Astronomical Observatory of Japan. For the radiation test, the FDCCD detector is irradiated with a proton beam at the Tandem van de Graff accelerator laboratory, Kyoto University. The
Nano-JASMINE project is a collaboration with the ISSL (Intelligent Space Systems Laboratory), University of Tokyo and is also supported by colleagues in the JASMINE working group.

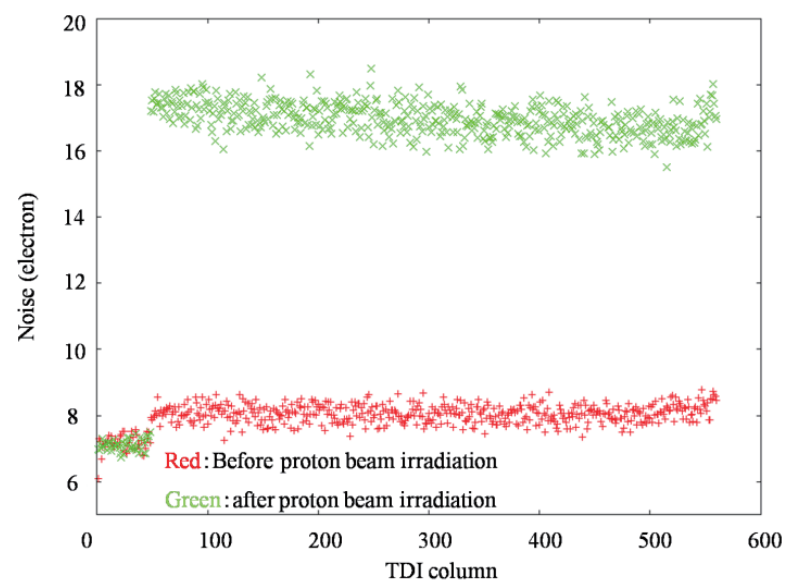

Fig. 5. The noise level of the FDCCD detector. Red and green points indicate the noise level before and after proton beam irradiation, respectively. We can see an overscan area where two noise levels correspond with each other, which are equal to a readout noise level.

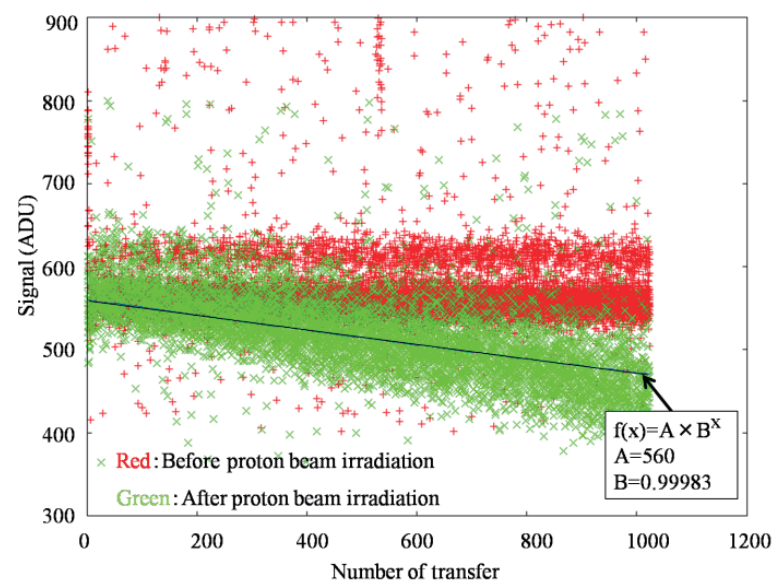

Fig. 6. CTE evaluation method by Fe-55 stacking. Red and green points indicate amount of signal charge for X-ray incidents before and after proton beam irradiation, respectively.

\section{References}

1) Kobayashi, Y., et al.: Nano-JASMINE: A 10-kilogram satellite for space astrometry, Proc. SPIE, 6265 (2006), pp. 626544 1-10.

2) Gouda, N.: Series of JASMINE missions, EAS Publications Series, 45 (2011), pp. 393-396.

3) Perryman, M., et al.: The hipparcos catalogue, Astronomy \& Astrophysics, 323 (1997), pp. 49-52.

4) Inamori, T., et al.: Attitude estimation using star images in Nano-JASMINE mission, Proceedings of the 27th ISTS, 2009-d-13 (2009).

5) Hatsutori, Y., et al.: Development of $5 \mathrm{~cm}$ Reflecting Telescope for Nano-JASMINE, Proceedings of the 27th ISTS, 2009-m-10 (2009).

6) Kamata, Y., et al.: Recent results of the fully-depleted back-illuminated CCD developed by Hamamatsu, Proc. SPIE, 
6276 (2006), pp. 62761 U 1-10

7) A. Marechal, A.: Etude des Effets Combine de la Diffraction et des Aberration Geometriques sur l'image d'un point Lumineux, Revne D'ptique, 26 (1947), pp.257-277.

8) G. R. Hopkinson et al.: Proton Effects in Charge-Coupled Devices, IEEE trans. nucl. sci., 43 (1996), 614.

9) http://www.spenvis.oma.be.

10) J. Janesick: Scientific Charged Coupled Devices, SPIE Press, (2001), pp. 132-139. 